J. Klin. Endokrinol. Stoffw. 2018 · 11:122-126 https://doi.org/10.1007/s41969-018-0050-7 Online publiziert: 27. November 2018 (c) Der/die Autor(en) 2018

CrossMark

\title{
SGLT-Hemmer als therapeutische Option bei Patienten mit Typ 1 Diabetes mellitus
}

verfügbaren Studien zeigen jedoch keinen überzeugenden Benefit [8-10].

Ergebnisse aus der REMOVAL-Studie, ein 36 Monate dauernder RCT $(n=438)$, in dem Metformin als Zusatztherapie zur Insulintherapie bei T1DM untersucht wurde, konnten weder im primären Endpunkt eine signifikante Reduktion der Intima-Media-Dicke der Carotis zeigen, noch konnten sich die anfänglich moderaten Hämoglobin A1c (HbA1c)-Verbesserungen (-0,24\%) über die Studiendauer halten [11].

In ADJUNCT ONE (52 Wochen, $n=1398)$ zeigte Liraglutide zwar gegenüber Placebo eine signifikante HbAlcVerbesserung, Gewichtsreduktion und Reduktion der Insulintagesdosis, allerdings war die Verbesserung des HbAlc sehr moderat (je nach Dosis des Glucagon-like Peptid-1-Rezeptor-Antagonisten [GLP1-RA] zwischen 0,15-0,2\% nach 52 Wochen), während das Risiko für Hypoglykämien und Hyperglykämien mit Ketoazidosen signifikant stieg, was dazu führte, dass Novo Nordisk die Zulassung für Liraglutide bei T1DM nicht weiter verfolgte [12].

Nachdem Sodium-Glucose-linkedTransporter(SGLT)-Hemmer (SGLTi) einen insulinunabhängigen Wirkmechanismus besitzen und zur vermehrten Ausscheidung von Glukose über den Harn führen, stellen sie auch eine mögliche Therapieoption in Ergänzung zur Insulintherapie bei T1DM dar. Der vorliegende Reviewartikel soll die aktuelle Studienlage bei T1DM und die möglichen Nebenwirkungen darstellen (Übersicht in - Abb. 1).

\section{Empagliflozin - EASE-2 und EASE-3}

Das EASE-Programm (Empagliflozin as Adjunctive to inSulin thErapy) bei Patienten mit Typ 1-Diabetes (T1DM) umfasste zwei multizentrische, randomisierte, doppelblinde, Placebo-kontrollierte Parallelgruppenstudien mit einmal täglicher oraler Empagliflozin-Dosierung über 52 Wochen (EASE-2) bzw. 26 Wochen (EASE-3). EASE-2 und EASE-3 untersuchten die Sicherheit und Wirksamkeit von Empagliflozin 10 und $25 \mathrm{mg}$ als Zusatztherapie zu einer intensivierten Insulintherapie bei T1DM, EASE-3 hatte einen zusätzlichen Arm mit Empagliflozin 2,5 mg.

Die 723 Patienten für EASE-2 bzw. 961 Patienten für EASE-3 wurden hauptsächlich in Europa und Nordamerika rekrutiert. Sie hatten eine Diabetesdauer von durchschnittlich 21,7 Jahren und einen durchschnittlichen Body-Mass-Index (BMI) von $\sim 28,6 \mathrm{~kg} / \mathrm{m}^{2}$.

Es zeigte sich eine HbAlc-Senkung zwischen $-0,45$ und $-0,54 \%$ mit den beiden höheren Empagliflozin-Dosierungen, während es unter Empagliflozin $2,5 \mathrm{mg}$ zu einer HbAlc-Reduktion um $-0,28 \%$ kam. Auch die Gewichtsreduktion fiel unter der 2,5-mg-Dosierung geringer aus (•Tab. 1). Die niedrige Dosierung zeigte jedoch im Gegensatz zu 10- oder 25-mg-Dosierung keine Erhöhung der Ketoazidose-Häufigkeit [13].

Die Autorinnen Caren Sourij und Anna Obermayer haben zu gleichen Teilen zum Manuskript beigetragen. 


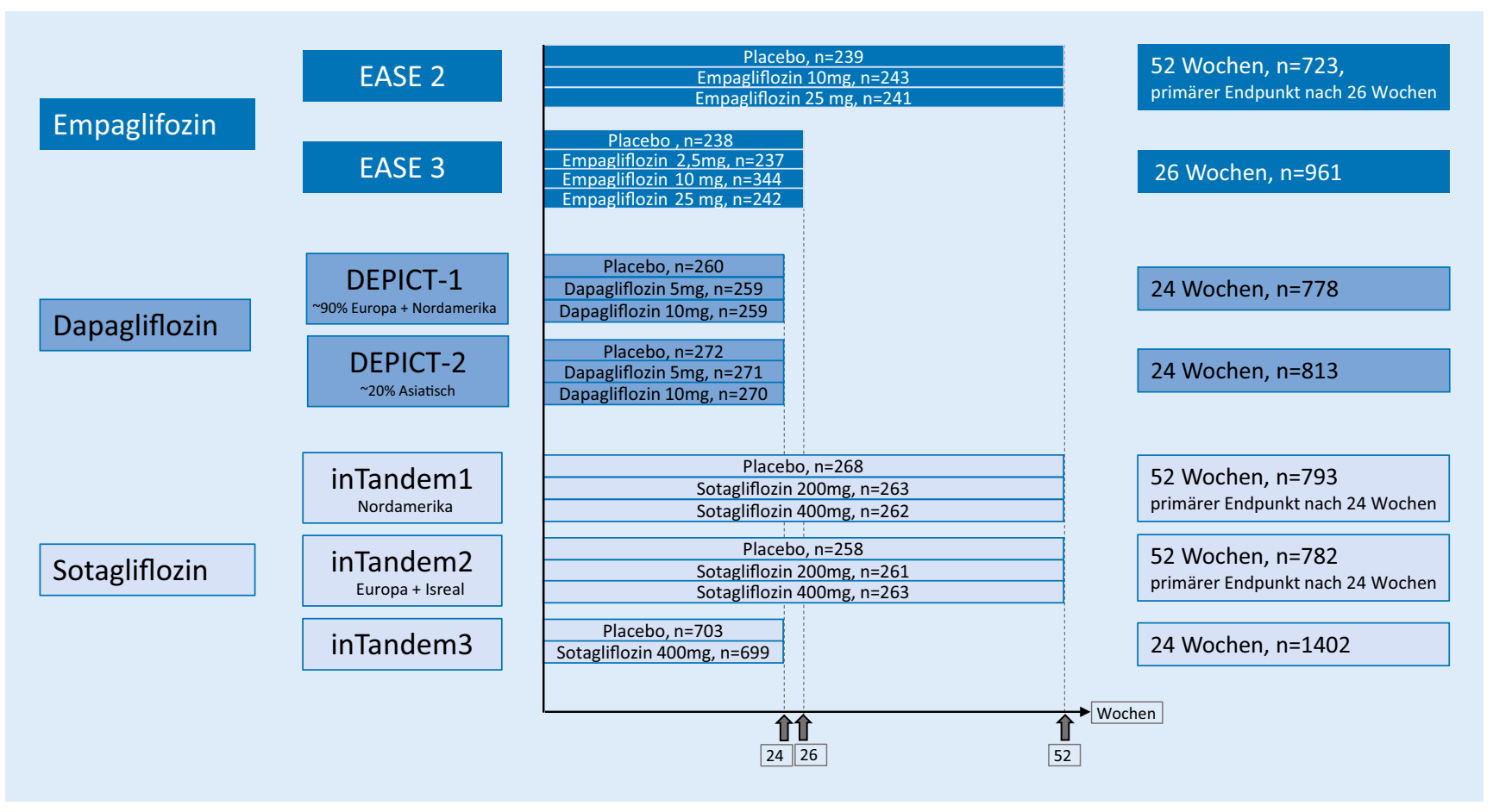

Abb. 1 \ Überblick über die Studien von Sodium-Glucose-linked-Transporter(SGLT) (1)/2-Hemmern bei Typ 1-Diabetes mellitus und deren wichtigsten Charakteristika

\section{Dapagliflozin - DEPICT-1 und DEPICT-2}

\section{DEPICT-1}

DEPICT-1 (Dapagliflozin Evaluation in Patients With Inadequately Controlled Type 1 Diabetes) untersuchte Dapagliflozin $5 \mathrm{mg}$ und $10 \mathrm{mg}$ im Vergleich zu Placebo für 24 Wochen als Zusatztherapie zu einer Insulintherapie bei Patienten mit unzureichend therapiertem T1DM.

Die 778 überwiegend europäischen StudienteilnehmerInnen hatten ein Durchschnittsalter von $42,5( \pm 13,9)$ Jahren und eine mittlere Diabetesdauer von 20,3 $( \pm 11,8)$ Jahren.

Sowohl die $5 \mathrm{mg}$ - als auch die $10 \mathrm{mg}$ Dosierungen von Dapagliflozin zeigten eine signifikante Reduktion des HbAlc ( $-0,42$ vs. $-0,45 \%)$ und des Körpergewichts $(-2,96 \%$ versus $3,72 \%)$. Die Raten an diabetischen Ketoazidosen lagen bei $2 \%$ (Dapagliflozin $5 \mathrm{mg}$ ) bzw. 3\% (Dapagliflozin $10 \mathrm{mg}$ ) [14].

\section{DEPICT-2}

DEPICT-2 war die zweite randomisierte Studie zur Bewertung der Wirksamkeit und Sicherheit von Dapagliflozin $5 \mathrm{mg}$ und $10 \mathrm{mg}$ für 24 Wochen als Zusatztherapie zu Insulin bei 813 erwachsenen Patienten mit Typ 1-Diabetes und inadäquater glykämischer Kontrolle. Während DEPICT 1 primär bei europäischen und nordamerikanischen StudienteilnehmerInnen durchgeführt wurde, wurden in DEPICT-2 auch $20 \%$ asiatische TeilnehmerInnen eingeschlossen.

Das Durchschnittsalter der Studienpopulation betrug 42,7 Jahre, mit einer Diabetesdauer von 19,3 Jahren. Der mittlere HbAlc-Ausgangswert betrug 8,4\%, das durchschnittliche Körpergewicht initial 79,2 kg, und der BMI-Ausgangswert war im Mittel 27,6 kg/m².

Die Studienergebnisse waren mit den Resultaten der DEPICT-1 Studie vergleichbar (• Tab. 1; [15]).

\section{Sotagliflozin - inTandem1, inTandem 2 und inTandem3}

Der duale SGLT 1- und SGLT 2-Hemmer Sotagliflozin wurde in Kombination mit Insulintherapie in den inTandem-Studien 1-3 untersucht.

\section{InTandem1}

Diese multizentrische, randomisierte, doppelblinde, Placebo-kontrollierte Studie, die an 75 Standorten in den USA und Kanada durchgeführt wurde, untersuchte die Sicherheit und Wirksamkeit von oralem Sotagliflozin 200 oder $400 \mathrm{mg}$ als Add-on-Therapie zu Insulin für 52 Wochen bei erwachsenen Patienten mit unzureichend therapiertem T1DM.

793 Patienten mit einer durchschnittlichen Diabetesdauer von $24,4( \pm 12,8)$ Jahren, einem mittleren HbA1c von $7,6 \%( \pm 0,7)$ und einem durchschnittlichen BMI von $29,7 \mathrm{~kg} / \mathrm{m}^{2}$ nahmen an der Studie teil.

Der HbAlc-Wert konnte durch die Einnahme von Sotagliflozin $200 \mathrm{mg}$ um $-0,36 \%$ und Sotagliflozin $400 \mathrm{mg}$ um $-0,41 \%$ reduziert werden. Das Körpergewicht nahm um $-2,79 \%$ bzw. um $-4,02 \%$ ab. Das Risiko einer diabetischen Ketoazidose zeigte sich bei Sotagliflozin $200 \mathrm{mg}(3,4 \%)$ und $400 \mathrm{mg}(4,2 \%)$ erhöht [16]. 


\section{InTandem2}

InTandem2 untersuchte die Gabe von einmal täglich Sotagliflozin 200 oder $400 \mathrm{mg}$ in Kombination mit einer optimierten Insulintherapie für 52 Wochen bei Erwachsenen mit T1DM.

782 Patienten mit einer durchschnittlichen Diabetesdauer von 18,4 Jahren $( \pm 10,9)$, einem mittleren HbAlc von $7,7 \%( \pm 0,8)$ und einem durchschnittlichen BMI von $27,8 \mathrm{~kg} / \mathrm{m}^{2}( \pm 5,1)$ wurden in 17 europäischen Ländern und Israel eingeschlossen.

Der HbAlc-Wert wurde unter Sotagliflozin $200 \mathrm{mg}$ um $-0,37 \%$ und unter 400 mg um $-0,35 \%$ reduziert. Das Körpergewicht der Patienten konnte unter $200 \mathrm{mg}$ um $-2,48 \%$ und unter $400 \mathrm{mg}$ um $-3,08 \%$ gesenkt werden. Das Risiko einer diabetischen Ketoazidose zeigte sich mit 2,3\% unter der $200 \mathrm{mg}$-Dosierung bzw. mit 3,4\% unter $400 \mathrm{mg}$ Sotagliflozin [17].

\section{InTandem3}

Über 24 Wochen wurden die 1402 TeilnehmerInnen mit T1DM entweder mit Sotagliflozin 400 mg oder Placebo behandelt.

Unter Sotagliflozin $400 \mathrm{mg}$ zeigte sich eine Reduktion des HbA1c um $-0,46 \%$ und des Körpergewichts um -2,98 kg, die Rate der diabetischen Ketoazidosen lag bei $3 \%$ [18].

In allen oben angeführten Studien zeigte sich unter dem SGLT-Hemmer kein erhöhtes Risiko für schwere Hypoglykämien [13, 15].

\section{Diskussion}

In allen Phase-3-Studien der genannten Programme konnte eine Senkung des HbAlc-Wertes, eine Senkung der Insulin-Tagesdosis sowie eine Gewichtsreduktion bei der Behandlung mit SGLTHemmern bei Patienten mit T1DM und unzureichender glykämischer Kontrolle gezeigt werden. Alle Studien haben Teilnehmer eingeschlossen, die im Mittel einen BMI zwischen 28 und $30 \mathrm{~kg} / \mathrm{m}^{2}$, hatten, auch wenn es nicht zwingend ein Einschlusskriterium war

Wie schon aus den Studien bei Typ 2 Diabetes mellitus bekannt, er- höhen SGLT-Hemmer auch bei T1DM das Risiko für genitale Pilzinfektionen. Während Dapagliflozin, Sotagliflozin und Empagliflozin 10 oder $25 \mathrm{mg}$ zwar eine akzeptable, blutzuckersenkende Potenz aufweisen, tritt unter allen eine bis zu 3-fach erhöhte Rate für diabetische Ketoazidosen auf. Für Empagliflozin 2,5 mg zeigt sich diese Erhöhung in EASE-3 zwar nicht, jedoch fällt die Blutzuckersenkung mit einer HbAlcReduktion von $-0,28 \%$ sehr moderat aus.

Nachdem SGLT-Hemmer den Blutzucker auch während einer Ketoazidose durch die weitere Ausscheidung der Glukose über den Harn im normoglykämischen Bereich halten (euglykämische Ketoazidose), werden Ketoazidosen unter SGLT-Hemmertherapie möglicherweise auch erst später erkannt, da mit der Hyperglykämie ein wesentliches Charakteristikum fehlt. Ketoazidosen bleiben weiterhin eine potenziell lebensbedrohliche Komplikation bei T1DM mit Letalitätsraten, die entsprechend rezenten US-Daten zwar insgesamt unter $1 \%$ liegen, jedoch deutlich höher bei Personen mit einem Alter über 60 Jahre [19] ausfallen. Eine mögliche Ursache für die erhöhte Rate an Ketoazidosen ist die gesteigerte Sekretion von Glukagon, die unter SGLT-Hemmern beobachtet wird. Dies könnte zu einem vermehrten Transport von Fettsäuren in die Mitochondrien und somit gesteigerter Betaoxidation führen [20]. Demnach kommt der richtigen Auswahl von potenziellen Patienten mit T1DM, die eine unterstützende SGLT (1)/2-Hemmertherapie erhalten sollen, sowie der Aufklärung über das richtige Verhalten im Rahmen von Erkrankungen eine wichtige Rolle zu. • Tab. 2 fasst die wichtigsten Maßnahmen im Rahmen eines solchen Therapiebeginns zusammen.

Derzeit laufen die Zulassungsverfahren für SGLT-Hemmer bei T1DM, es bleibt aber noch zu definieren, welche Patientengruppen besonders davon profitieren können. Dies werden in erster Linie übergewichtige oder adipöse Patienten mit T1DM sein, bei denen mit einer Insulintherapie trotz Optimierungsversuchen keine zufriedenstellende Blutzuckereinstellung erreicht werden kann. Ob SGLT-Hemmertherapien abseits der
J. Klin. Endokrinol. Stoffw. 2018 11

122-126

https://doi.org/10.1007/s41969-018-0050-7

(c) Der/die Autor(en) 2018

\section{Sourij · A. Obermayer $\cdot$ H. Sourij}

SGLT-Hemmer als therapeutische Option bei Patienten mit Typ 1 Diabetes mellitus

\section{Zusammenfassung}

Während eine Insulintherapie bei Diabetes mellitus Typ 1 überlebensnotwendig ist, werden unterstützende antihyperglykämische Substanzen untersucht, die mithelfen können, die glykämische Kontrolle zu erleichtern, ohne das Hypoglykämierisiko zu steigern. Natrium-Glukose-Cotransporter (SLGT)-Hemmer führen zu einer Glukosurie und damit verbundener Blutzuckersenkung, ein Mechanismus, der auch bei Typ 1 Diabetes mellitus potentiell erfolgversprechend ist. Der vorliegende Review fasst die aktuellen Effizienzdaten bei Verwendung eines SGLT-Hemmers bei Typ 1 Diabetes zusammen und beleuchtet aber auch das Risiko der euglykämischen Ketoazidose, welches mit dieser Therapie assoziiert ist

\section{Schlüsselwörter}

Dapaglifozin · Empaglifozin · Insulin . Ketoazidose $\cdot$ Sotaglifozin

\section{SGLT-inhibitors as a therapeutic option for patients with type 1 diabetes}

Abstract

While insulin therapy is vital for subjects with type 1 diabetes, supporting glucose lowering agents are needed, helping to optimize glycaemic control while not increasing hypoglycaemia risk. Sodium glucose co-transporter (SGLT)-inhibitors are lowering blood glucose by increasing glucosuria, a mechanism also working in type 1 diabetes. This review summarizes current efficacy data of SGLT-inhibitors in type 1 diabetes and discusses the increased risk for euglycaemic ketoacidosis associated with this drug class.

Keywords

Dapaglifozin · Empaglifozin · Insulin .

Ketoacidosis · Sotaglifozin 


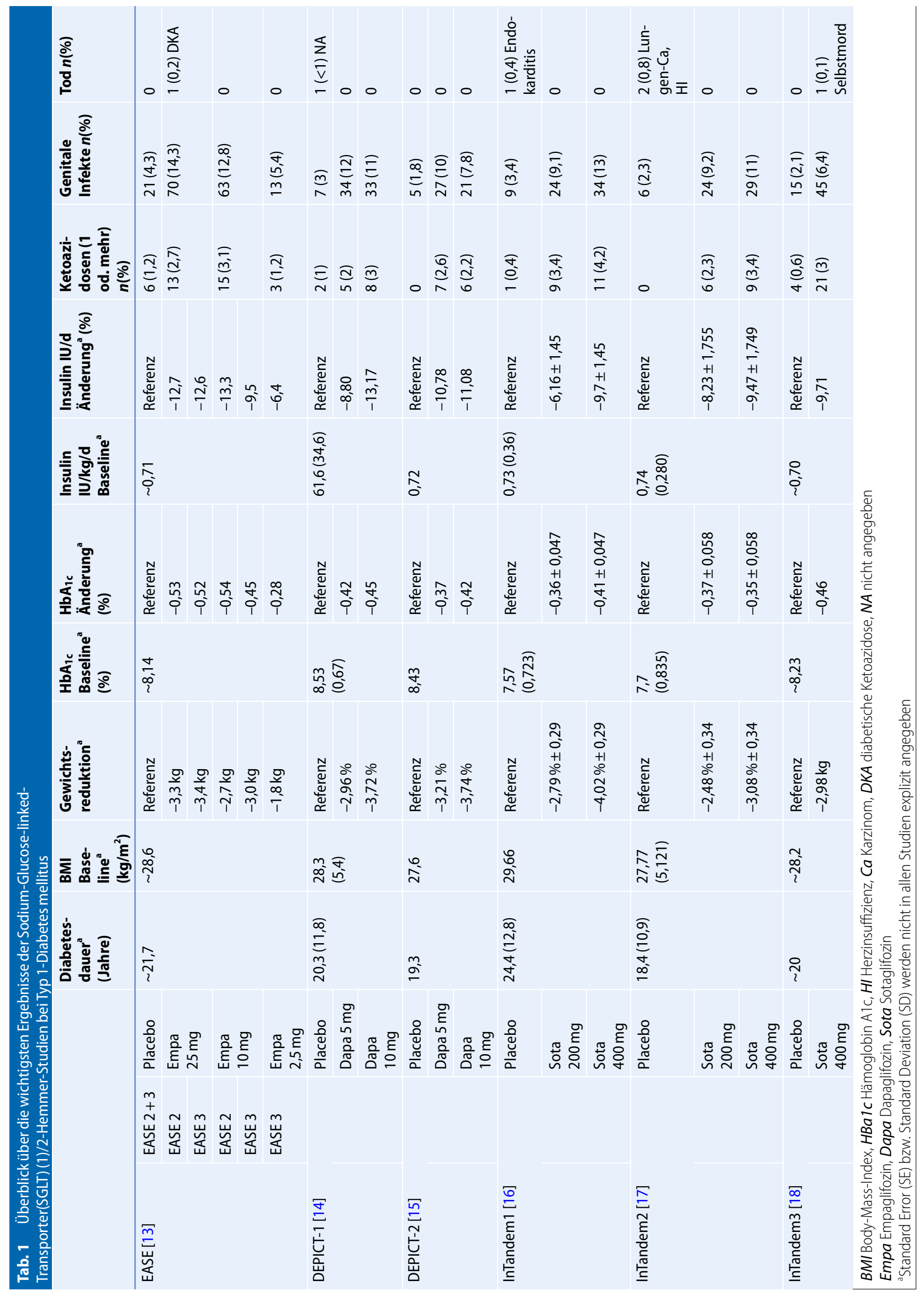


Tab. 2 Empfohlene Maßnahmen beim Einsatz von SGLT (1)/2-Hemmer bei Typ 1-Diabetes mellitus. (Adaptiert nach [21])

\section{Empfehlungen für eine zusätzliche SGLTi-Therapie bei Patienten mit T1DM}

Eine Therapie mit SGLTi ist nicht ratsam, wenn Patienten eine eingeschränkte Compliance zeigen, einen $\mathrm{HbA} 1 \mathrm{c}-$ Wert $>75 \mathrm{mmol} / \mathrm{mol}$ (9\%) haben oder eine Ketoazidose in der Anamnese haben Berücksichtige das erhöhte Risiko einer Ketoazidose bei Patienten mit Insulinpumpentherapie Verwende die niedrigste Dosis von SGLTi, die nötig ist, um einen klinischen Benefit zu erreichen Reduziere das prandiale Insulin anfangs um 10-20\% und titriere je nach prä- und postprandialen Blutzuckerwerten

Reevaluiere die Insulin-Kohlenhydrat-Ratio (den Korrekturfaktor) bei bestehender SGLTi-Therapie Gib dem Patienten ein Blut-Ketonkörpermessgerät mit und kläre den Patienten bezüglich Ketoazidosen und deren Management auf

Empfehle das Testen auf Blut-Ketonkörper, wenn sich der Patient krank fühlt und der Blutzucker $>160 \mathrm{mg} / \mathrm{dl}$ ist

Empfehle das Absetzen der SGLTi-Therapie während eines Krankheitsbildes, das viel Flüssigkeitsverlust oder geringe Flüssigkeitszufuhr mit sich bringt

Kläre den Patienten über das erhöhte Risiko einer genitalen Pilzinfektion (und Diarrhoe) auf

SGLTi Sodium-Glucose-linked Transporter Inhibitor, HbA1c Hämoglobin A1c

Blutzuckersenkung auch bei T1DM protektive renale und/oder kardiovaskuläre Effekte aufweisen, müsste in gesonderten Endpunktstudien untersucht werden.

\section{Korrespondenzadresse}

\section{Assoz. Prof. PD Dr. Harald Sourij}

Klinische Abteilung für Endokrinologie und Diabetologie, Medizinische Universität Graz Auenbruggerplatz 15, $8036 \mathrm{Graz}$, Österreich ha.sourij@medunigraz.at

Funding. Open access funding provided by Medical University of Graz.

\section{Einhaltung ethischer Richtlinien}

Interessenkonflikt. C. Sourij und A. Obermayer geben an, dass kein Interessenkonflikt besteht. H. Sourij erhielt unrestricted research grants von Astra Zeneca und Böhringer Ingelheim und erhielt Sprecherhonorare Astra Zeneca und Böhringer Ingelheim.

Dieser Beitrag beinhaltet keine von den Autoren durchgeführten Studien an Menschen oder Tieren.

Open Access Dieser Artikel wird unter der Creative Commons Namensnennung 4.0 International Lizenz (http://creativecommons.org/licenses/by/4.0/deed. de) veröffentlicht, welche die Nutzung, Vervielfältigung, Bearbeitung, Verbreitung und Wiedergabe in jeglichem Medium und Format erlaubt, sofern Sie den/die ursprünglichen Autor(en) und die Quelle ordnungsgemäß nennen, einen Link zur Creative Commons Lizenz beifügen und angeben, ob Änderungen vorgenommen wurden.

\section{Literatur}

1. Nathan DM, Genuth S et al (1993) The effect of intensive treatment of diabetes on the development and progression of long-term complications in insulin-dependent diabetes mellitus. N Engl J Med 329(14):977-986. https:// doi.org/10.1056/NEJM199309303291401

2. Nathan DM, Cleary PA, Backlund JY et al (2005) Intensive diabetes treatment and cardiovascular disease in patients with type 1 diabetes. N Engl J Med 353(25):2643-2653. https://doi.org/10.1056/ NEJMoa052187

3. The Diabetes Control and Complications Trial Research Group (1997) Hypoglycemia in the Diabetes Control and Complications Trial. Diabetes 46(2):271-286 (http://www.ncbi.nlm.nih.gov/ pubmed/9000705)

4. The DCCT Research Group (1988) Weight gain associated with intensive therapy in the diabetes control and complications trial. Diabetes Care 11(7):567-573. https://doi.org/10.2337/diacare. 11.7.567

5. McKnight JA, Wild SH, Lamb MJE, Cooper MN, Jones TW, Davis EA et al (2015) Glycaemic control of Type 1 diabetes in clinical practice early in the 21st century: an international comparison. Diabet Med 32(8):1036-1050 (http://www.ncbi.nlm.nih. gov/pubmed/25510978)

6. Miller KM, Foster NC, Beck RW, Bergenstal RM, DuBoseSN, DiMeglio LA etal (2015) Current state of type 1 diabetes treatment in the U.S.: updated data from the T1D Exchange Clinic Registry. Diabetes Care 38(6):971-978 (http://www.ncbi.nlm.nih. gov/pubmed/25998289)

7. George P, McCrimmon RJ (2013) Potential role of non-insulin adjunct therapy in type 1 diabetes. Diabet Med 30(2):179-188. https://doi.org/10. 1111/j.1464-5491.2012.03744.x

8. Griffin KJ, Thompson PA, Gottschalk M, Kyllo JH, Rabinovitch A (2014) Combination therapy with sitagliptin and lansoprazole in patients with recent-onset type 1 diabetes (REPAIR-T1D): 12-month results of a multicentre, randomised, placebo-controlled, phase 2 trial. Lancet Diabetes Endocrinol 2(9):710-718
9. Hari Kumar KVS, Shaikh A, Prusty P (2013) Addition of exenatide or sitagliptin to insulin in new onset type 1 diabetes: a randomized, open label study. Diabetes Res Clin Pract 100(2):e55-e58. https:// doi.org/10.1016/j.diabres.2013.01.020

10. Zhao Y, Yang L, Xiang Y, Liu L, Huang G, Long Z et al (2014) Dipeptidyl peptidase 4 inhibitor sitagliptin maintains $\beta$-cell function in patients with recentonset latent autoimmune diabetes in adults: one year prospective study. J Clin Endocrinol Metab 99(5):876-880

11. Petrie JR, Chaturvedi N, Ford I, Brouwers MCGJ, Greenlaw N, Tillin T et al (2017) Cardiovascular and metabolic effects of metformin in patients with type 1 diabetes (REMOVAL): a doubleblind, randomised, placebo-controlled trial. Lancet Diabetes Endocrinol 5(8):597-609. https://doi. org/10.2337/dc16-0691

12. Mathieu C, Zinman B, Hemmingsson JU, Woo V, Colman P, Christiansen E et al (2016) Efficacy and safety of Liraglutide added to insulin treatment in type 1 diabetes: the ADJUNCT ONE treat-to-target randomized trial. Diabetes Care 39(10):1702-1710 (http://www.ncbi.nlm.nih.gov/ pubmed/27506222)

13. Rosenstock J, Perkins BA, Marquard J, Laffel LM, Neubacher D, Kaspers S et al (2018) Empagliflozin as adjunctive to insulin therapy in type 1 diabetes: the EASE trials. Diabetes Care. https://doi.org/10 2337/dc18-1749

14. Dandona P, Mathieu C, Phillip M, Hansen L, Griffen SC, Tschöpe D et al (2017) Efficacy and safety of dapagliflozin in patients with inadequately controlled type 1 diabetes (DEPICT-1): 24 week results from a multicentre, double-blind, phase 3 , randomised controlled trial. Lancet Diabetes Endocrinol 5(11):864-876

15. Mathieu C, Dandona P, Gillard P, Senior P, Hasslacher C, Araki Eet al (2018) Efficacy and safety of Dapagliflozin in patients with inadequately controlled type 1 diabetes (the DEPICT-2 study): 24-week results from a randomized controlled trial. Diabetes Care. https://doi.org/10.2337/dc180623

16. Buse JB, Garg SK, Rosenstock J, Bailey TS, Banks P, Bode BW et al (2018) Sotagliflozin in combination with optimized insulin therapy in adults with type 1 diabetes: the North American Intandem 1 Study. Diabetes Care. https://doi.org/10.2337/ dc18-0343

17. Danne T, Cariou B, Banks $P$, Brandle $M$, Brath $H_{t}$ Franek E et al (2018) HbA1c and hypoglycemia reductions at 24 and 52 weeks with Sotagliflozin in combination with insulin in adults with type 1 diabetes: the European Intandem2 Study. Diabetes Care. https://doi.org/10.2337/dc18 0342.

18. Garg SK, Henry RR, Banks P, Buse JB, Davies MJ, Fulcher GR et al (2017) Effects of Sotagliflozin added to insulin in patients with type 1 diabetes. N Engl J Med. https://doi.org/10.1056/NEJMoa1708337

19. Umpierrez G, Korytkowski M (2016) Diabetic emergencies-ketoacidosis, hyperglycaemic hyperosmolar state and hypoglycaemia. Nat Rev Endocrinol 12(4):222-232. https://doi.org/10. 1038/nrendo.2016.15

20. Ogawa W, Sakaguchi K (2016) Euglycemic diabetic ketoacidosis induced by SGLT2 inhibitors: possible mechanism and contributing factors. J Diabetes Investig 7(2):135-138

21. Mccrimmon RJ, Henry RR (2018) SGLT inhibitor adjunct therapy in type 1 diabetes. Diabetologia 61:2126-2133 